Cinémas

Revue d'études cinématographiques

Journal of Film Studies

\title{
Les limbes du scénario
}

\section{Gilles Thérien}

Volume 9, numéro 2-3, printemps 1999

Les Scénarios fictifs

URI : https://id.erudit.org/iderudit/024789ar

DOI : https://doi.org/10.7202/024789ar

Aller au sommaire du numéro

\section{Éditeur(s)}

Cinémas

\section{ISSN}

1181-6945 (imprimé)

1705-6500 (numérique)

Découvrir la revue

Citer cet article

Thérien, G. (1999). Les limbes du scénario. Cinémas, 9(2-3), 103-121.

https://doi.org/10.7202/024789ar

\section{Résumé de l'article}

Parler de scénario fictif, c'est évoquer un texte au statut « trouble ». S'il existe seul, sans sa source, s'il est sa propre source, ou encore s'il ne s'est jamais réalisé dans un film, il demeure un texte qu'on ne sait comment lire. C'est un texte de nature transitive et, pour décider de sa valeur, il faut évaluer comment s'opère le passage entre la source, événement ou fiction, et sa réalisation, un film. C'est le cas qui est développé dans cet article. Comment passer de la lecture à la « spectature "? Comment respecter les deux médias impliqués sans avoir à inventer une position neutre où tout pourrait se retrouver, s'étudier? La question est d'autant plus difficile que le transit s'opère à partir du domaine privé de la lecture vers le domaine public de la «spectature ». Les Fous de Bassan, roman d'Anne Hébert et le film d'Yves Simoneau qui porte le même titre servent d'exemples. 


\section{Les limbes du scénario}

\section{Gilles Thérien}

\section{RÉSUMÉ}

Parler de scénario fictif, c'est évoquer un texte au statut "trouble". S'il existe seul, sans sa source, s'il est sa propre source, ou encore s'il ne s'est jamais réalisé dans un film, il demeure un texte qu'on ne sait comment lire. C'est un texte de nature transitive et, pour décider de sa valeur, il faut évaluer comment s'opère le passage entre la source, événement ou fiction, et sa réalisation, un film. C'est le cas qui est développé dans cet article. Comment passer de la lecture à la "spectature » ? Comment respecter les deux médias impliqués sans avoir à inventer une position neutre où tout pourrait se tetrouver, s'étudier? La question est d'autant plus difficile que le transit s'opère à partir du domaine privé de la lecture vers le domaine public de la "spectature». Les Fous de Bassan, roman d'Anne Hébert et le film d'Yves Simoneau qui porte le même titre servent d'exemples.

\section{ABSTRACT}

The idea of fictitious screenplay involves the notion of an unstable status of the text. Whether it exists in itself, without its source, or is its own source, or has never been realized into a film, it is a text whose reading remains problematic. As a transitive text, an assessment of its value depends on the movement from the source (event or fiction) to its realization as film. This is the case study presented in this article. How does the shift from reading to "spectating" happen? How is it possible to respect the two media involved without having to come up with a neutral position where everything could meet and be studied? The question is all the more difficult since the shift occurs between the private sphere of reading and the public sphere of 
"spectating." Les Fous de Bassan, a novel by Anne Hébert as well as a film by Yves Simoneau, will serve as examples.

Parler de scénario fictif, c'est évoquer un texte au statut "trouble". S'il existe seul, sans sa source, s'il est sa propre source, ou encore s'il ne s'est jamais réalisé dans un film, il demeure un texte qu'on ne sait comment lire. Faut-il le lire en fonction de sa source et poser des questions de fidélité ou le lire en fonction d'un film qui n'existe pas et parler alors de son infaisabilité? On pourrait aussi poser la question de la lecture du texte même du scénario: comment donne-t-il à voir? On connaît enfin la scénarisation qui se présente comme un transit vers l'œuvre romanesque. Il suffit de citer le plan de L'Antiphonaire d'Hubert Aquin pour comprendre qu'une telle chose puisse exister, mais il faut bien comprendre qu'à mesure que le roman s'écrit, le «scénario" s'efface.

Dans son sens le plus traditionnel, le scénario est le texte à partir duquel des décisions de production peuvent être prises. Il peut contenir diverses sortes de documents compte tenu du type de film envisagé. L'objectif de son écriture est de donner à voir. C'est la première phase dans l'élaboration de l'objet visuel. Lorsque le film est terminé, le scénario n'a plus sa raison d'être. Il peut devenir une archive du film et servir aux études cinématographiques ou encore, il peut accéder à une nouvelle carrière sous forme de publication de scénario construit à partir du film déjà réalisé et faisant disparâttre tout ce qui, dans le scénario original, permet de suivre le processus de réalisation. Ces publications sont surtout utiles comme accompagnement du film, mais elles présentent en regard de ce dernier un aspect nettement répétitif, ce qui est rarement le cas dans les scénarios véritables. Lorsque le film n'est pas réalisé, le scénario devient alors une sorte de texte "stérile" parce qu'il se donne sous l'angle de la lecture et non de la spectature. Il permet de suivre le contexte de certaines décisions de production, mais ne dit rien de ce vers quoi le scénario lui-même tendait.

On peut facilement comprendre la nature transitive du scénario et pour décider de sa valeur, il faut voir les relations 
auxquelles il est soumis: par rapport au roman qui est à sa source ou par rapport au film qui en est le produit. Or c'est dans le transit que s'opère le passage entre la source, événement ou fiction, et sa réalisation, un film. C'est le cas qui nous intéresse ici. Comment passer de la lecture à la spectature? Comment respecter les deux médias impliqués sans avoir à inventer une position neutre où tout pourrait se retrouver, s'étudier? La question est d'autant plus difficile que le transit s'opère à partir du domaine privé de la lecture vers le domaine public de la spectature. Ce que nous souhaitons discuter ici, c'est la difficulté de ce passage et les problèmes qui en découlent. Le scénario est responsable de la façon dont le transit doit s'effectuer. Je prendrai comme exemple Les Fous de Bassan, le roman d'Anne Hébert et le film d'Yves Simoneau (1996) qui porte le même titre et qui se présente comme une adaptation du roman. Dans des perspectives de lecture et de spectature, je m'attacherai à l'examen de trois cas : le point de vue du roman, celui du film et la coprésence de la lecture et de la spectature.

Le roman d'Anne Hébert, Les Fous de Bassan, est publié au Seuil en 1982. Il se compose de six sections dont les formes d'écriture changent selon le contexte de la section. Deux sections, la première et la dernière, sont datées de l'automne 1982; il s'agit du livre de Nicolas Jones et de la dernière lettre de Stevens Brown adressée à Michael Hotchkiss au même automne 1982. Une troisième section s'intitule "Olivia de la Haute Mer" et est «sans date \%. Elle précède la dernière lettre de Stevens. Les trois autres sections sont, dans l'ordre, les lettres de Stevens Brown à Michael Hotchkiss, le livre de Nora Atkins et celui de Perceval Brown et de quelques autres. Les trois datent de l'été 1936. Le récit s'ouvre sur une mise en place des lieux: la mer à perte de vue, les ruines du village de Griffin Creek, le village multicolore qui le remplace et le presbytère de Nicolas Jones auquel on a ajouté une galerie des ancêtres. C'est de son presbytère que parle le pasteur Jones, un soir d'automne, discours embué de la furnée des pipes qui se succèdent et des réminiscences qui l'empêchent de dormir. Avec sa galerie des ancêtres et les deux jumelles Brown, le "vieillard perclus" offre ses rêves, des cauchemars plutôt, qui mettent en relation historique 
1782 et l'automne de 1982. Ce bicentenaire macabre raconte la chute et la ruine d'une petite communauté de loyalistes anglais chassés de Nouvelle-Angleterre au moment de la signature du traité de Versailles et venus se réfugier plus au Nord dans ce pays qui appartient à la couronne britannique, le Canada. On pourra remarquer au passage qu'Anne Hébert semble situer le traité de Versailles, dont elle ne parle pas nommément, en 1782 alors qu'il est signé un an plus tard, en 1783. Les loyalistes ont-ils fui à l'avance ou Anne Hébert qui publie en 1982 a-t-elle voulu protéger sa propre date de publication? Cela n'a pas, en apparence, une grande importance sauf si l'on porte, comme il se doit, un soin particulier au travail de lecture.

Grâce aux diverses notions développées depuis les beaux jours du structuralisme, il serait possible de se poser diverses questions sur le texte. Qui est le narrateur? Est-il singulier ou multiple? Comment s'établit la diégèse, quelles sont les principales situations qui marquent son développement? De la même façon, on peut comparer les personnages entre eux, évaluer leurs actions, identifier les divers sujets d'énonciation. Toutes ces questions ont pour effet de ramener la lecture du récit à se définir comme une histoire qui peut être reprise dans ses objectifs comme dans son déroulement, à se limiter à marquer la forme argumentative qu'adopte ce récit. La question devient alors très simple. À l'été 1936, Stevens Brown, absent de son village depuis cinq ans à la suite d'altercations violentes avec son père, revient et retrouve le village. Il demeure un garçon farouche et violent, mais il se laisse attirer par deux jeunes filles, les cousines Atkins. Le village vit durant l'été des moments pénibles, soit autour du suicide de la femme du pasteur, soit au moment de la disparition des deux cousines Atkins dans la nuit du 31 août. Stevens est soupçonné, traduit en justice, puis libéré. Il participera ensuite à la Seconde Guerre mondiale, reviendra détraqué et vivra désormais à Montréal, à l'hôpital des vétérans. Un jour, il s'enfuit et écrit une longue et dernière lettre à son ami américain Michael Hotchkiss qu'il n'a pas revu depuis 1936 et dont il ne sait s'il est mort ou vivant. Il lui raconte sa culpabilité dans le meurtre des deux cousines en même temps qu'il lui annonce la décision de mettre fin à ses jours. On peut ajouter 
ou supprimer des détails. L'essentiel est là au plan de l'argumentation. Il reste à savoir pourquoi cela met 250 pages à s'écrire si la chose est si simple.

Ce qui est écarté dans ce qui précède, c'est le travail de lecture qui consiste à mettre en évidence la ou les figures qui, entre les lignes, au-delà de la forme argumentative, maintiennent la relation au texte. Une figure se met en place dès le début du texte. En effet, il existe un lieu où tout va se nouer et se dénouer, un lieu à partir duquel la chute d'un groupe de familles et d'individus est inévitable. Le pasteur Nicolas Jones qui, normalement, se présente comme un obstacle à cette chute, apparaît plutôt comme un de ses acteurs et non des moindres. Sa vieillesse, à la lecture du livre qui porte son nom, est marquée par l'échec devant Dieu et devant les hommes. Cet échec est celui d'un paradis perdu. Le peuple élu de la colonie de Nouvelle-Angleterre est expulsé de son paradis terrestre pour être ensuite forcé de se réfugier chez les papistes, c'est-à-dire chez ceux avec qui aucun commerce n'est possible, les catholiques francophones. La petite communauté de Griffin Creek se développera dans les marges de l'inceste et dans les méandres du mal. Ce que le pasteur rumine à travers les volutes de la fumée de ses multiples pipes, c'est la culpabilité qui hante toute sa communauté. Les petites Atkins, il les a, lui aussi, désirées, palpées du regard ou des mains comme dans le cas de Nora; d'autres que lui ont eu les mêmes sentiments. Griffin Creek, c'est la conjonction de la grandeur et de la petitesse. Cette dernière va gagner parce qu'elle est la réduction extrême de l'espace laissé aux loyalistes. Perceval, le jeune et le fou, est aussi impliqué par une débilité congénitale; il la verra prendre de plus en plus de place chez lui, jusqu'à l'internement. D'une certaine façon, les deux jeunes filles sont elles-même coupables parce que, au sortir de l'adolescence, elles ont voulu, vite, le plus vite possible, être des femmes. Quand, à la toute fin du roman, Stevens, devenu lui aussi malade mental, s'accuse des meurtres à partir des circonstances qu'il est le seul à connaître, il n'en décrit pas rnoins l'état délabré de son esprit maintenant comme à l'époque. Sa vie, celle où il a peut-être connu un certain moment de bonheur, est marquée par un séjour de cinq ans aux États-Unis, surtout en Floride, période dont on sait bien peu de 
choses sinon qu'elle apparaît comme un retour temporaire au paradis. Au moment même où il vit dans le passé, le pasteur exerce son autorité malveillante sur les deux sœurs jumelles de Stevens et de Perceval. En somme, chaque moment du roman est teinté de culpabilité. Il est aussi balayé par la mer, les grands vents, les cris furieux des fous de Bassan. La nature représentée par la mer est celle qui donne la folie et la mort. C'est auprès d'elle que les passions s'exacerbent. Seule Felicity Jones, la mère du pasteur, semble échapper à cette mer inexorable peut-être parce que Felicity, qui initie ses petites filles Nora et Olivia au contact de la mer, vit dans les deux mondes, celui de l'exil et de la ruine comme celui de la folie et de la mort. La lecture du roman fait apparaître cette figure de la culpabilité comme horizon et comme condition de vie. La condamnation précède les actes et les suit. Nul n'échappe à ce destin de l'expulsion du paradis.

Dans une pareille perspective, on doit se demander comment le travail de lecture parvient à faire apparaître ces figures et à les maintenir parallèlement et au-delà de l'argumentation narrative. Le lecteur au contact du texte et dans le cours de sa lecture met en place ses propres images mentales, celles qui appartiennent à son contexte de lecture, à ses attitudes culturelles. En même temps qu'il lit, il travaille à la construction de la memoria, du topos romanesque que l'imagination va féconder. La figure peut ainsi être validée tant du côté de l'angle intime de l'acte que du côté de sa confrontation à la culture dont elle s'inspire. En lisant, je construis les représentations mentales essentielles à la vie de ce que je lis, à ma participation à cette vie plutôt qu'au jeu stérile d'y chercher un sens prétendument caché. Pendant que je lis, le jeu des représentations mentales me permet de suivre avec souplesse la direction de la lecture. L'investissement imaginal que je fais dans le texte permet la naissance de la figure, ici celle de la culpabilité qui suit la chute, celle, au fond d'un péché originel qui se charge d'incarner dans des destins particuliers un des mythes les plus importants de notre culture judéo-chrétienne. Mon appréciation du roman est faite de cette "découverture", de cette construction qui donne à ce qu'on peut alors nommer une œuvre d'art, sa raison d'être, sa "suffisance". 
Le régime des images mentales qui accompagnent la lecture s'inaugure à partir de ma conscience de lecteur. C'est dire que je ne peux lire les divers moments des livres séparément les uns des autres. Ils font tous partie d'un même réseau unifié par ma conscience. Par exemple, je ne peux séparer dans ma conscience les personnages les uns des autres. Des points d'ancrage me permettent de les identifier: leur nom, le sexe auxquels ils appartiennent, leur âge, leur rôle social. Et, c'est sur la base de ces diverses données que je peux suivre un personnage en le regardant évoluer au milieu des autres personnages et dans les diverses situations romanesques. Je n'ai pas besoin de tout savoir de ce personnage, un noyau d'éléments stables suffira à ma capacité de reconnaître et de distinguer. Prenons comme exemple Stevens Brown, celui par qui le mal s'accomplit.

La première mention de Stevens Brown apparaît dans la section du roman qui a pour titre «Le Livre du révérend Nicolas Jones, automne 1982". Elle nous livre son prénom, sa qualité de frère des jumelles qui vivent sous la coupe du pasteur: "Non complètement idiotes comme leur frère Perceval, ni maléfiques comme leur autre frère Stevens [...]» (p. 17). Un peu plus loin, c'est, à partir du nom de famille, un résumé fracassant d'un des enjeux du récit: "John et Bea Brown ayant mis au monde Stevens, Perceval et les jumelles, s'en sont débarassés, au cours d'un seul été" (p. 21). Puis le narrateur Nicolas Jones trace en quelques mots le portrait de Stevens Brown:

Lui, à contre-jour, campé sur ses longues jambes, dans l'encadrement de la porte, silhouette sombre dégingandée et résolue, nimbée de soleil, de la tête aux pieds, se refusant à entrer, se refusant à être un des nôtres, se refusant à partager avec nous les chants et la prière. Son visage émacié, ses yeux pâles dans l'ombre de son chapeau marron (p. 28).

C'est la description matricielle du personnage du jeune Stevens; elle est donnée tout d'un coup. Elle sera reprise, modulée, mais demeure inchangée tout au long du roman jusqu'à la dernière section formée par la lettre de Stevens Brown alors âgé de 66 ans, enfermé dans une chambre de fortune en train d'écrire une lettre en forme de testament. Notons ici les traits qui servent à décrire le 


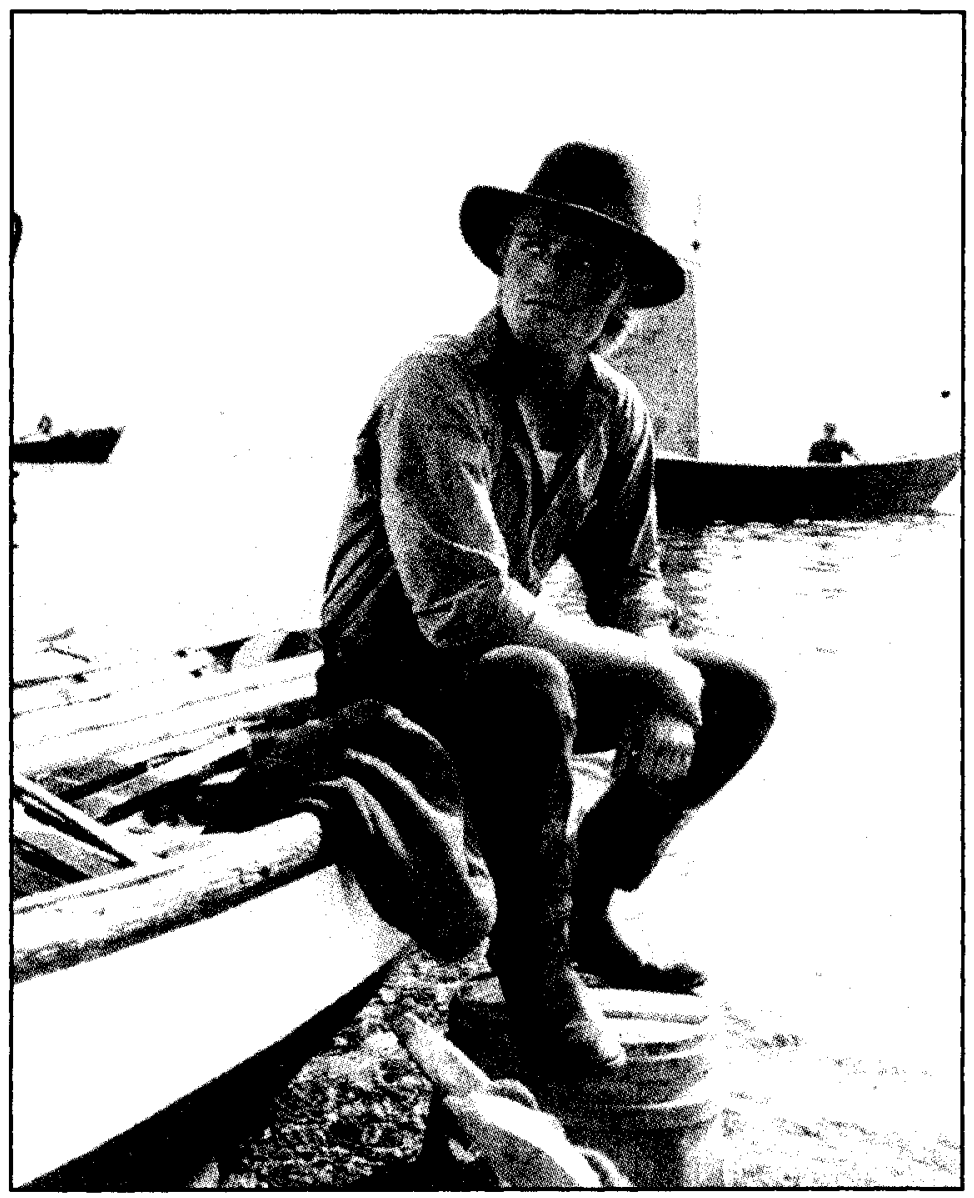

\section{Les Fous de Bassan d'Yves Simoneau (1986)}

personnage. C'est d'abord une silhouette, "la ligne générale d'un corps" comme le dit le dictionnaire Larousse. C'est une enveloppe, un contenant, sombre au demeurant et, en ce sens, difficile à décrire avec précision. S'il a de longues jambes, l'ensemble est pourtant dégingandé c'est-à-dire "disloqué dans ses mouvements et dans sa démarche" - toujours le dictionnaire! Le nimbe du soleil l'entoure comme une auréole. Son chapeau mou cache mal un visage émacié et des yeux pâles. Sa première apparition publique a lieu dans l'embrasure de la porte demeurée ouverte de la petite église de Griffin Creek au moment où tout le village y est 
rassemblé pour écouter la parole de Dieu transmise par Nicolas Jones. Cette description vient incarner le nom de Stevens Brown, fils d'une famille pour le moins étrange, prête à exploser comme la violence incontrôlée du père. La seule autre mention descriptive du personnage dans cette section est liée à la danse à laquelle assiste tout le village, Stevens compris, danse qui sert de prélude au suicide d'Irène Jones, l'épouse stérile du pasteur: "Stevens a gardé son chapeaui sur la tête, ce qui ne l'empêche pas de swinguer avec entrain. Ses longues jambes bougent en mesure, comme électriques, endiablées" (p. 47). Les cinquante-quatre premières pages ont campé le personnage. La suite des sections donnera des précisions sur ces traits qui sont déjà bien définis, mais, en gros, c'est la redondance qui domine. De temps en temps, une barbe de deux à trois jours donne au jeune Stevens un air malpropre et peu respectable; il est "[...] chaussé de bottes viriles, été comme hiver, le chapeau vissé sur la tête, ne se découvrant ni pour l'église ni pour les femmes" (p. 61), ce qui ne l'empêche pas, en se décrivant à son correspondant Michael Hotchkiss (été 36), de se donner un double:

Encore un peu de temps et vous me verrez, encore un peu de temps et vous ne me verrez plus. Rien à faire pour éviter la comparaison, trop de lectures bibliques, dans morı enfance sans doute, si quelqu'un ressemble au Christ dans ce village, c'est bien moi, Stevens Brown. Non tant à cause de la barbe de trois jours et du chapeau, enfoncé sur les yeux, mais plutôt à cause de mon état de passage à Griffin Creek (p. 88-89).

Quand il visite ses parents, il est rasé et ses cheveux sont taillés. Il n'en garde pas moins son chapeau sur la tête. Enfin, un détail qui oscille entre l'accessoire et l'objet intime, il lui arrive de jouer de l'harmonica, ce qui lui permet de créer un lien avec Perceval et avec Nora. Ces traits de Stevens sont décrits du point de vue de Nicolas Jones et participent à un double emploi, celui de décrire le personnage une fois pour toutes et celui de fournir au lecteur l'interprétation de Jones qui écrit ses souvenirs, lui aussi, en 1982.

Dans le "Livre de Nora", les traits demeurent largement les mêmes si ce n'est qu'ils prennent pour la narratrice une 
coloration plus négative: "[..] sa personne aux jambes interminables [...] son maigre visage, ses yeux pâles et perçants [...]. Le beau visage dur de Stevens, ses longues jambes, son sexe de garçon, caché dans ses habits de garçon, son mépris [...]" (p. 127) et d'ajouter Nora, "ma rage" parce qu'elle désire Stevens qui ne lui retourne pas son désir. Mais on aura tout de même noté la convergence des traits.

Dans le "Livre de Perceval", des changements importants apparaissent: "[...] ses yeux déteints [...] le creux de ses joues [...]" (p. 150), "Mon frère Stevens n'a plus de chapeau" (p. 158), et le passage où tout bascule:

Mon frère Stevens ouvre la porte de la chambre. S'appuie au chambranle. Se met en marche dans la cuisine. Avec peine. Comme s'il cherchait ses pas à mesure. Se demande chaque fois quel pied il va poser par terre. Ça ne ressemble pas à mon frère Stevens. Sans regard. Ni fierté. Les traits tirés. Les jambes et les bras trop longs. L'air égaré. Il boit à la pompe de la cuisine. Il met son visage sous l'eau. Pompe à tour de bras. Se relève. S'essuie la figure, le cou et les cheveux avec son mouchoir. La voix forte de McKenna appelle dans la pièce à côté. - Reviens ici. J'ai encore quelques petits détails à te demander. Mon frère Stevens retourne dans la chambre, l'air d'un chien qu'on tire par sa laisse et qui se résigne mal à obéir (p. 191-192).

C'est qu’à partir de maintenant, on a franchi le cap des événements mystérieux de la nuit du 31 août 1936. Perceval décrit la suite en témoin qui aime son frère et qui ne comprend pas ou ne veut pas comprendre le changement qui s'est opéré chez lui. De dégingandé et résolu qu'il était au moment de son retour, Stevens n'a gardé que la dislocation de la démarche et de l'âme.

Olivia, dans son rôle de revenante de la Haute Mer, reprend des détails qui apparaissent pour la première fois et qui trahissent son amour pour Stevens, un amour déjà ancien puisqu'il remonte à l'enfance (p. 206) sans pour autant modifier la description matricielle: "La chaleur de son corps tout près du mien. Son odeur de tabac et d'alcool. Ses yeux en vrille sous l'ombre de son chapeau [...]» (p. 202), "Il roule des épaules en marchant et ses yeux sont couleur de cendres bleues" (p. 214), 
"C'est Stevens qui m'a touchée, lui qui a un petit cal à la main droite" (p. 219) ou encore, à propos de l'épisode de la tempête entre le 20 et le 31 août: "Le voici avec eux émergeant de la tempête, l'œil rouge, les vêtements trempés, ivre d'alcool et de visions marines [...] son ceil fou [...] cette voix pâteuse [...] lui qui titube et pue l'alcool $[. .$.$] » et "Le lendemain Stevens, hâve$ et les yeux couleur d'étoupe, parvient à tirer de sa cervelle brumeuse le seul souvenir vivace de sa nuit obscure. Il dit que toute la nuit il s'est senti appelé, tandis que sur son éperon de rocher il subissait l'assaut des éléments déchaînés et que la tempête cognait contre ses tempes [...]" (p. 221). De la fameuse nuit du 31, elle nous laisse quelques détails, la chaleur de son bras et, surtout, "[...] ses lèvres [qui] se retroussent sur ses dents en un sourire étrange» (p. 224). On remarque que, pour Olivia, les traits passent d'un pôle à l'autre: le regard devient fou, la chaleur du corps s'accompagne d'une odeur forte, le sourire paraît étrange. Et les deux ont en commun cette difformité presque secrète, le cal de la main et le doigt palmé.

Le roman nous projette ensuite 46 ans plus tard. Stevens, dans la soixantaine, hospitalisé depuis 37 ans au Queen Mary, écrit à son habituel destinataire Hotchkiss, mort ou vivant, et qu'il imagine vieux, lui aussi : "Old, tu dois l'être pour de vrai à présent, sans doute bedonnant, du moins flasque, les petites rides du rire, au coin de tes yeux, creusées comme des sillons" (p. 229). N'est-ce pas dans un miroir qu'écrit Stevens, n'est-ce pas de lui-même qu'il parle ici, de sa propre déchéance corporelle? Les détails qu'il donne sur sa propre vie ajoutent à ce qui pourrait se voir :

Mes blessures de guerre ne sont pas visibles à l'œil nu [...]. La face intacte [...] plutôt blanche [...] l'arête du nez bien dessinée [...]. Je ne suis pas malade. Je pleure et je crie. Je tremble et je frissonne [...]. Pas la moindre petite cicatrice. Détraqué seulement. Complètement détraqué (p. 230-231).

En expliquant ce qui s'est passé entre Nora, Olivia et lui, les seuls détails qu'il précise sont l'étranglement de Nora, le viol, suivi de l'étranglement d'Olivia, ses mains et la pression des 
doigts autour des cous, et son sexe, comme une "arme qui bande». Les mains et les doigts sont des traits nouvellement marqués.

Cette description suit le déroulement de la lecture. Comme nous l'avons déjà dit, le personnage est mis en scène à partir d'une description très économique. La silhouette au départ, "dégingandée et résolue», contient déjà ce qui fonde les deux versants du drame: la résolution qui l'habite complètement, de la tête aux pieds, et la dislocation qui viendra s'y substituer à partir de la tempête qui précède le fatidique 31 août déjà en germe dans la tare héréditaire qui unit les habitants de Griffin Creek depuis 1782. L'acte de lecture du personnage Stevens Brown s'appuie sur cette matrice qui sert à représenter le personnage: le nom propre, Stevens Brown, son sexe et son statut parental et les traits descriptifs physiques déjà identifiés, soit les jambes longues, l'allure, le regard, le cal de la main, etc. L'acte de lecture prête au personnage une conscience, la conscience du lecteur, qui vient fournir l'élément essentiel du développement du personnage, l'intentionnalité. C'est cette dernière qui articule la description au nom. C'est elle aussi qui permet aux divers traits de converger et de maintenir la substance du personnage tout au long de la lecture.

Cette figure du personnage n'est qu'une sorte d'horizon intellectuel, plus ou moins vide, qui n'engage aucune reconnaissance visuelle précise chez le lecteur. On pourra tout au mieux parler d'image mentale. Le lecteur demeure libre d'imaginer les personnages comme il l'entend. Mais, en même temps, la relation établie par le lecteur avec le personnage assume connaissance et reconnaissance d'où cette impression de familiarité que procure la lecture. On pourrait reprendre chaque personnage et faire le même exercice. On se rendrait alors compte de l'immense liberté d'imaginer dont jouit le lecteur. Il peut suppléer à ce qu'il ne trouve pas dans le texte à la seule condition de porter attention à la convergence des traits et à leur permanence. Il en va tout autrement dans le cas d'un film où l'imagerie mentale est remplacée par de véritables images.

Ce qui importe ici, c'est de voir d'abord comment le film présente la même matière imaginale transcrite dans un système 


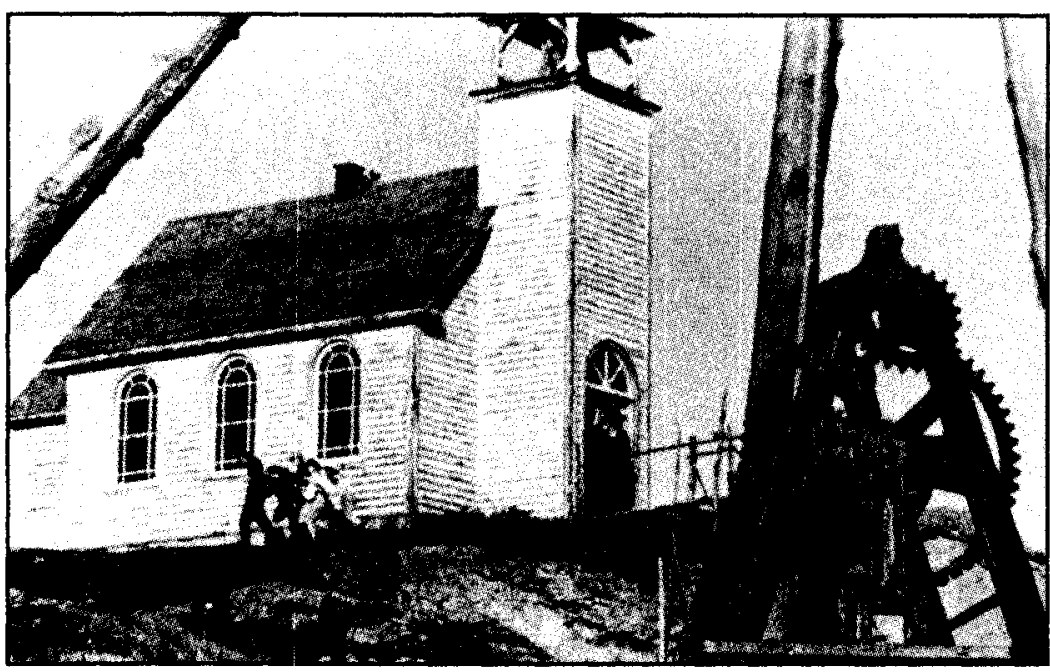

\section{Les Fous de Bassan d'Yves Simoneau (1986)}

que nous nommerons, pour l'instant, l'illusion de ressemblance. Le matériau source du film est le même roman, mais contentonsnous, dans un premier temps, de regarder la transcription du personnage de Stevens. J'ai choisi d'examiner les tout premiers plans du film. Ces plans restent d'ailleurs dans une forme de générique allongé pendant lequel les principales données de l'histoire sont mises en place.

L'image n'apparaît qu'avec le titre. Il s'agit d'un bord de mer, le soir. Au loin, on distingue une silhouette claire qui suit le bord de mer en direction de la caméra placée plus ou moins dans l'axe du spectateur. Un gros plan d'une jeune fille en insert coupe ce déroulement. La jeune fille répète: "N'y va pas, Olivia, n'y va pas!» On reprend la marche d'Olivia en plan moyen, puis, en plongée... Olivia s'approche d'une forme disloquée étendue sur le rivage, à la frontière de la mer et de la plage. On dirait un cadavre rejeté par la mer. Olivia s'en approche, secoue la personne qui, finalement, reprend vie ou s'éveille et se tourne vers Olivia. Il s'agit d'un jeune homme. On voit son visage en gros plan, rasé et moúillé. Personne ne parle. Il cherche à s'emparer d'Olivia. Jeu de regards qui exprime la résistance, le refus. Puis il s'empare d'elle et roule avec elle sur la plage. On entend les cris de la jeune fille. La scène est violente, 
mais vue de loin. Réapparition muette de l'autre jeune fille en insert pendant la scène et les cris. On change alors de séquence. Il fait jour. On voit la mer au loin. Une voix monologue « Tout le monde sur l'île est d'accord pour dire qu'il n'y avait pas de vent $[. .$.$] " pendant que la caméra suit la main du personnage,$ puis son bras et enfin l'ensemble du haut de sa personne que l'on découvre. À ce moment, il dit: "Moi, Stevens Brown [...]. "On le voit ensuite en plan américain, en vêtements amples, qui se dirige vers une porte ouverte sur l'extérieur. Il tourne le dos à la caméra. Dehors, il coiffe un chapeau mou. On passe à un gros plan du jeune homme de la scène précédente, cadré de façon à couper le dessus de sa tête chevelue, suivi, dans un faux contrechamp, d'un gros plan de la tête chauve de Stevens Brown âgé. À ce moment, la voix de Stevens Brown âgé dit: "Il ne me reste qu'une image déformée de moi-même qu'à revoir, me revoir comme j'ai toujours voulu me voir, moi, Stevens Brown." Suit un dernier retour au gros plan du jeune homme avant le changement de séquence.

Ces deux longues séquences nous semblent suffisantes pour illustrer ce que nous voulons dire de la figuration iconique. On voit bien ici comment le processus est différent. Des personnages sont d'abord donnés dans la totalité ou dans la plus grande partie de leur matérialité corporelle. Par exemple, ici, dans la première séquence, on ne voit pas tout très clairement, mais on voit les personnages suffisamment longtemps pour les identifier. Dans la seconde séquence, on ne voit jamais, par exemple, les jambes de Stevens Brown âgé ni la totalité du corps du jeune Stevens. La représentation du personnage est totalement différente. Parler alors d'un script visuel des personnages, c'est faire appel à une situation immensément complexe parce que le donné du personnage repose sur un nombre très élevé de traits fixes. On ne peut se contenter d'une silhouette, il faut voir en détail le visage, les yeux, la bouche, la forme du nez, les cheveux, etc., et cela ne suffit pas, il y a aussi le regard, le ton de la voix, le débit... et, du corps entier, il faut saisir le mouvement, la démarche, le maintien, le costume qui vient de façon si particulière marquer les personnages pour qu'on puisse les reconnaître. Il n'y a pas ici de case vide laissée à l'imagination, 
mais bien une case pleine qui doit, dès le départ, contenir tous les éléments qui serviront par la suite. Le noyau de base, qui était une structure plus souple dans le domaine de la représentation mentale discursive, est ici de l'ordre de l'objet singulier. Il s'agit d'une personne qui est sa propre référence. Reconnaître le personnage, c'est comme reconnaître quelqu'un sur la rue. Contrairement à ce qui se produit dans la représentation discursive, je n'ai pas ici à projeter sur lui une conscience et une intentionnalité, il y a relation visuelle et auditive entre le spectateur et le personnage. Toute la gamme de la séduction s'inscrit dans cette relation. Le cinéma comme illusion de la vie donne aussi l'illusion de la conscience des personnages, mais il oblige le spectateur à inférer, à partir des gestes et des paroles, une interprétation de cette intentionnalité. Or quel moyen en propre possède le cinéma pour assurer la convergence de tous les traits d'un personnage en fonction de son développement cohérent dans le récit filmique? Aucun. Il faut se fier au choix du comédien, à la distribution, au casting des comédiens qu'il vaudrait mieux traduire par «moulage», car c'est bien de cela qu'il s'agit : il faut créer un moule unique du personnage pour le rendre crédible, plus vrai que vrai. Le spectateur est mis en demeure de tisser avec les divers personnages des relations affectives qu'il projette selon ce qu'il perçoit et à partir de ses propres affinités. La direction artistique, les costumes et les maquillages, la mise en scène ont leur part dans la réussite de cette projection, mais seulement à la condition que le personnage soit déjà habité, comme au théâtre, par le comédien.

L'examen de la représentation du personnage au cinéma indique bien la nature de la différence entre la lecture et la spectature. Le noyau stable est constitué d'un personnage singulier, la personne du comédien, ce qui comprend son caractère, c'est-à-dire son nasque au sens théâtral du terme. L'évolution du personnage est marquée, elle, par les costumes et les accessoires qui décrivent l'aspect privé du personnage. Les jeux de rôles ne peuvent apparaître que dans la mesure où un personnage est confronté à d'autres personnages, ce qui constitue habituellement une trame importante des films. On peut penser à la difficulté qu'il y a à caractériser un très grand nombre de 
personnages ce qui fait, par exemple, du Bal d'Ettore Scola (1984) un véritable tour de force.

Regardons de plus près comment le film Les Fous de Bassan réalise concrètement le transit sur le personnage de Stevens Brown entre le roman et le film. Il faut deux comédiens parce qu'il est impossible de rajeunir l'un ou de vieillir l'autre suffisamment pour rendre crédible le personnage. Il ne peut y avoir continuité des traits. Il faut donc littéralement deux personnes pour un même nom. Le film choisit de les disposer dans deux séquences consécutives. C'est d'abord le jeune Stevens qui nous est présenté mais, de façon morcelée, le corps allongé, recroquevillé, de loin, des profils où on voit sa figure mouillée, ses cheveux lissés par la pluie où on sent une calvitie naissante. Il ne parle pas. Un jeu de regards fort discutable sur le plan de la mise en scène du viol finit par retrouver un sens autour de l'œil globuleux du personnage âgé. De cette séquence sombre, on passe à une séquence de plein jour. L'autre personnage est donné progressivement. Le morcellement est ici une procédure de découverte et non de fragmentation. En outre, c'est le vieux Stevens Brown qui parle et qui parle d'une chose passée, d'une chose qu'il reste encore à expliquer et, en parlant, il prend soin de se nommer, de se désigner. C'est lui qui a la parole. Pourtant presque immédiatement, le gros plan du jeune Stevens Brown s'interpose, toujours muet. Il est ensuite lié au plan de Stevens Brown âgé au moment où ce dernier affirme: "Il ne me reste qu'une image déformée de moi-même à revoir, me revoir comme j'ai toujours voulu me voir, moi, Stevens Brown. "Il faut, semble-t-il, prendre cela au pied de la lettre. Ce qui est vu est aussi une image déformée dans un sens comme dans l'autre. Le spectateur est alors requis de transférer sur le jeune homme le nom propre qu'il avait déjà identifié au plus vieux. Le retour du gros plan du jeune Stevens vient renforcer le processus. En outre, l'ensemble de la séquence, toute tournée vers une réinterprétation du passé, intègre la première séquence dans la seconde comme elle avait intégré les plans du jeune Stevens dans ceux du vieux. La boucle est bouclée, le spectateur est averti, le générique peut se terminer et le film commencer avec l'histoire de Stevens Brown jeune. On pourra noter avec plaisir comment certains éléments de la lecture du roman viennent s'installer dans le film, la présence de 
la main, l'air "christique» du jeune Stevens, l'allure dégingandée et résolue et le corps recroquevillé de la séquence du début, mais on notera aussi comment le personnage n'a aucune trace des tares héréditaires qui sont présentes un peu partout dans le roman. Le transit, s'il est acceptable au niveau des personnages, se passe à un autre niveau.

Au cinéma, la figuration iconique qui est donnée demeure dans le monde de la singularité comme le monde dont il est le simulacre. Ce qu'il faut alors produire, ce sont des inférences appliquées à la représentation discursive, à partir de la figuration iconique. Or cette dernière est, la plupart du temps, hautement codée et oblige le spectateur au choix de certaines inférences. Par exemple, une image qui contient la tour Eiffel est un stéréotype de la France. Le spectateur est alors amené à conclure que l'action se passe en France. En fait, le cinéma est un vaste répertoire de ces interprétances stéréotypées, interprétances qui ne valent que pour des objets singuliers, mais qui permettent des énoncés soumis à des lois de véridiction plutôt qu'à un régime de vraisemblance. C'est qu'en fait le travail d'organisation narrative au cinéma ne se fait pas dans le film, mais doit être inféré par le spectateur à partir des données filmiques. Le cinéma, sauf exception, laisse moins de choix au spectateur que la littérature. Il bloque, pendant son déroulement, le phénomène de l'imagerie mentale et impose au spectateur des déroulements logiques qu'il ne peut suggérer que par la cohérence des images. C'est dire que plus un roman engage une lecture fine, peu figurative et ouverte, moins il est susceptible d'être adapté au cinéma... et plus un roman engage une lecture simple, de type inférentiel certains romans policiers par exemple - , plus il est facile à adapter. En somme, le transit du roman au cinéma se passe à des niveaux différents. Du côté romanesque, c'est le travail de visualisation mentale, impliqué par la lecture, qui apparaît comme une tâche ultérieure. Au cinéma, c'est le travail d'organisation narrative qui est non plus impliqué, mais plutôt inféré par le déroulement des séquences. Le premier processus tend à replacer le roman dans le monde de la singularité; le second amène le film au niveau d'une représentation discursive, diégétique, de la représentation visuelle. 
Il ne sera donc pas étonnant de voir jusqu'à quel point, même au plan d'un bref schéma narratif, le film s'éloigne du roman. Stevens Brown âgé est devenu peintre. C'est lui qui, en quelque sorte, remplace ses sœurs jumelles qui habitent avec Nicolas Jones. Il est loin du personnage damné du roman. Il ressuscite sous une forme un peu cynique, mais qui écarte la figure de la culpabilité personnelle et collective filée à travers les générations comme dans le roman. Des situations narratives nouvelles apparaissent comme l'étranglement du lapin par Stevens ou l'épisode de la chasse. L'accent est nettement mis sur l'aspect anecdotique du crime et de la violence qui l'accompagne. On pourrait ainsi poursuivre le déroulement du film sans jamais trouver de connivence figurale entre le roman et le film.

Le scénario est absent de notre réflexion. Il est peut-être dans les archives. Mais, à partir du moment où je me trouve en présence du roman et du film, je n'ai plus besoin du scénario. Le transit est opéré, mal peut-être, mais il l'est et le scénario ne servirait qu'à faire porter le blâme des décisions du film sur les éléments de production, de réalisation, de direction artistique... ou même sur la décision de faire une adaptation cinématographique de ce roman.

En fait, nous nous trouvons au cour même du problème: ce que donne à lire le roman dans le travail de lecture et ce que donne à voir le film dans le travail de la spectature. Nous dépassons alors le cas de notre exemple qui est loin d'être unique. La lecture se doit de faire vivre le texte en l'habitant imaginalement, c'est-à-dire en construisant la memoria qui sert de topos à l'action romanesque et en s'assurant que cette memoria est habitée par les principaux personnages. Dans pareil cas, c'est le lecteur qui prend en charge la dimension profonde de la relation imaginaire qu'il crée tout au long de sa lecture. Il singularise l'ouvre par cette prise en charge et la complexifie par sa capacité de contextualiser culturellement cette aventure. Il est, par exemple, plus difficile de complexifier les textes d'une culture qui nous est totalement inconnue. La spectature est différente. Un monde d'objets iconiques est fourni, une illusion du réel est mise en place. Le principe de référence dans la spectature dépend beaucoup de la cohérence des images. Le spectateur se 
voit, lui, incapable d'en fournir. Son rôle change alors. Il lui revient de donner au film la dimension de profondeur qui est absente en surface. C'est par ses propres projections affectives et imaginaires que le spectateur est amené à voir, dans le film, les figures qui échappent à la représentation visuelle des objets. S'il n'y parvient pas, c'est peut-être que l'on se trouve devant un film sans profondeur, tout en surface. Ou encore, c'est que, pour des raisons de censure personnelle, le spectateur refuse de se laisser aller à l'enjeu de la spectature. Dans cette opération, le scénario est disparu.

Il reste un cas à discuter, c'est celui où on voit le film d'abord et on lit le roman ensuite. Ce n'est certes pas l'idéal parce que les images fournies par le film vont venir faire obstruction à la création d'images mentales personnelles. La confusion des deux médias peut empêcher alors un travail de lecture satisfaisant. Cela dit, mieux vaut lire que pas du tout!

Le travail de scénarisation, on l'aura compris, est une lourde tâche qui exige le remplacement de la lecture par la spectature chez le ou la scénariste. Le premier transit doit avoir lieu là. On ne peut se fier à ce niveau à une transposition d'une histoire dans une autre, d'un fait narratif discursif par un fait narratif visuel. C'est se refermer sur la forme et collaborer à la réduction de l'œuvre. C'est créer une continuité illusoire entre deux médias. Des films comme Prospero's Books (1995) et Pillow-Book (1991) de Peter Greenaway n'ont en apparence que peu à voir avec l'œuvre de Shakespeare ou de Sei Shônagon et, pourtant, ils témoignent d'une aventure figurale qui leur est propre. C'est, me semble-t-il, ce que tout scénario doit chercher à faire transiter entre lecture et spectature: le fantôme qui donne la vie à la fiction.

\title{
Université du Québec à Montréal
}

\author{
OUVRAGE CITÉ
}

Hébert, Anne. Les Fous de Bassan. Paris: Le Seuil, 1982. 